\title{
Truncated first moment of the parton distribution - a modified approach
}

\author{
Dorota Kotlorz $\quad$ Andrzej Kotlorz ${ }^{\dagger}$
}

August 30, 2006

\begin{abstract}
We derive the LO DGLAP evolution equation for the full Mellin moments of the truncated at $x_{0}$ first moment of the nonsinglet parton distribution. This "moment of moment" approach allows to determine the small- $x_{0}$ behaviour of the truncated first moment. We compare our predictions to results obtained from $x$-space solutions for parton distributions with use of the Chebyshev polynomial method and to solutions of the evolution equations for the truncated moments proposed by other authors. The comparison is performed for different input parametrisations for $10^{-5} \leq x_{0} \leq 0.1$ and $1 \leq Q^{2} \leq 100 \mathrm{GeV}^{2}$. We give an example of application to the determination of the contribution to the Bjorken Sum Rule.

PACS 12.38.Bx Perturbative calculations, 11.55.Hx Sum rules
\end{abstract}

\section{Introduction}

Deep inelastic scattering (DIS) experiments provides knowledge about the internal structure of the nucleon. Measurements on proton, deuteron and neutron targets allow also verification of sum rules e.g. [1 2 basic relations in QCD. Sum rules for unpolarised and polarised structure functions refer to their Mellin moments. Particularly important role in the experimental and theoretical QCD tests play first moments of the parton densities, which have physical interpretation and can be determined from the data. From an experimental point of view, however, the accurate verification of sum rules is unreliable. Determination of the sum rules requires knowledge of the structure functions over the entire region of the Bjorken variable $x \in(0 ; 1)$. The lowest limit of $x$ in present experiments is about $10^{-5}$ and the limit $x \rightarrow 0$, which means that the invariant energy $W^{2}$ of the inelastic lepton-hadron scattering becomes infinite $\left(W^{2}=Q^{2}(1 / x-1)\right)$, will never be attained. Therefore it is very hopeful in the theoretical analysis to deal with truncated instead of with full moments of the structure functions. This enables to avoid uncertainties from the unmeasurable $x \rightarrow 0$ region. The most familiar theoretical approach, which describes scaling violations of parton densities in perturbative QCD has been formulated by Dokshitzer, Gribov, Lipatov, Altarelli and Parisi (DGLAP) 3. Evolution equations for the truncated at low $x_{0}$ moments of parton distributions are however more complicated than in a case of the full moments. These are not diagonal and each $n$-th truncated moment couples to $(n+j)$-th $(j \geq 0)$ truncated moments [4. For $n \geq 2$ the series of couplings to higher moments is very fast convergent. Even for small $(m=4)$ number of terms in the expansion of the truncated counterpart of the anomalous dimension $G_{n}$, the higher moments can be calculated with excellent accuracy. First moment is more sensitive to the truncated point $x_{0}$ and the convergence of $G_{n}$ for $n=1$ is weaker than for the higher moments. Nevertheless, it has been shown in [5], that for more terms of the $G_{n}$ expansion $(m \sim 30)$, the uncertainty in the determination of the first moment at $x_{0} \leq 0.1$ and $Q^{2}=10 \mathrm{GeV}^{2}$ does not exceed $5 \%$ independently on the input parametrisation. However, this increase with $m$ of the accuracy does not proceed infinitely. Numerical errors, which occur for larger $m$ (dependently on $x_{0}$ ) make further improvement of the precision impossible. It may be very useful to discuss methods of theoretical determination of truncated first moments of parton distributions because these predictions would be directly verified experimentally. In this paper we present an approach, in which we compute the truncated at small $x_{0}$ first moments using the inverse Mellin transform of their full moments. In other words, our method is based on the solutions for the full $n$-th moments of the truncated first moment of the parton distribution. This "moment of moment" technique would be a complementary one to other known methods in determination of sum rule contributions.

The content of this paper is as follows. In Section 2 we recall the ways of computing the truncated moments within DGLAP approximation. Thus Sect. 2.1 contains a brief description of the Chebyshevpolynomial approach for $x$-space solutions of DGLAP evolution equations. In Sect. 2.2] we recall the idea of DGLAP evolution equation for the truncated moments, which underlies our "moment of moment" technique. This modified method is presented in Sect.2.3 For simplicity we consider the quark nonsinglet LO evolution.

*Opole University of Technology, Division of Physics, Ozimska 75, 45-370 Opole, Poland, e-mail: dstrozik@po.opole.pl

$\dagger$ Opole University of Technology, Division of Mathematics, Luboszycka 3, 45-036 Opole, Poland, e-mail: kotlorz@po.opole.pl 
In Section 3 we compare solutions for the truncated at small $x_{0}$ first moment of the nonsinglet structure function, obtained with use of these mentioned earlier approaches. As an example we explore the Bjorken Sum Rule (BSR). In Section 4 we present predictions for the low- $x$ contribution to the BSR together with experimental constraints. Finally, in Section [5] we summarise our results.

\section{Determination of the truncated Mellin moments of parton distributions.}

For (full) Mellin moments of parton distributions $p\left(x, Q^{2}\right)$

$$
M[p]\left(n, Q^{2}\right)=\int_{0}^{1} d x x^{n-1} p\left(x, Q^{2}\right)
$$

the DGLAP evolution equation can be solved analytically. This is because in the moment space $n$ one obtains simple diagonalised differential equations. The only problem is the knowledge of the input parametrisation for the whole region $0 \leq x \leq 1$, what is necessary in the determination of the initial moments $M[p]\left(n, Q^{2}=Q_{0}^{2}\right)$ :

$$
M[p]\left(n, Q_{0}^{2}\right)=\int_{0}^{1} d x x^{n-1} p\left(x, Q_{0}^{2}\right) .
$$

Using the truncated moments approach one can avoid the uncertainties from the region $x \rightarrow 0$, which will never be attained experimentally.

The truncated at $x_{0}$ Mellin moment of the parton distribution $p\left(x, Q^{2}\right)$ is defined as

$$
M[p]\left(x_{0}, n, Q^{2}\right)=\int_{x_{0}}^{1} d x x^{n-1} p\left(x, Q^{2}\right) .
$$

From the theoretical point of view, there are two ways to avoid the problem of dealing with the unphysical region $x \rightarrow 0$. The first one is to work in $x$-space and obtain directly the evolution of parton distributions (not of their moments). The most known methods for solving the $Q^{2}$ evolution equations for parton distributions in $x$-space are brute-force [6], Laguerre-polynomial [7] or Chebyshev-polynomial [8 approaches. In this way, the truncated moment can be simply found by integrating the $x$-space solutions $p\left(x, Q^{2}\right)$ over the cut range $x_{0} \leq x \leq 1$ (see Sect. 2.1). An alternative way is use the evolution equations directly for truncated moments. The appropriate DGLAP evolution equations for the truncated moments have been derived in 4]. Authors have shown that these equations, though not diagonal, can be solved with a quite good precision for $n \geq 2$, even for a very small number of terms in the expansion series. In a case of the first moment, the accuracy is worse and more terms in the $G_{n}$ expansion must be taken into account. We briefly recall the idea of solving the evolution equations for truncated moments in Sect. 2.2 Basing on this idea, we have derived the evolution equation for truncated first moment in a diagonal form. The appropriate integro-differential equation contains only one function $-q_{1}\left(x, Q^{2}\right)$, which denotes truncated at $x$ first moment, without coupling to the other, higher moments. Then, using the full Mellin moments approach, we have found the small$x=x_{0}$ behaviour of the function $q_{1}\left(x, Q^{2}\right)$, which is simply the truncated at low- $x_{0}$ first moment. Detailed description is given in Sect. 2.3

\subsection{LO DGLAP evolution equations with use of the Chebyshev- polyno- mial expansion.}

The Chebyshev polynomials technique 9] was successfully used by J.Kwieciński in many QCD treatments e.g. 8]. Using this method one obtains the system of linear differential equations instead of the original integro-differential ones. For example, in order to solve the LO DGLAP evolution equation for the nonsinglet parton distribution $p \equiv q^{N S}$ :

$$
\frac{\partial p(x, t)}{\partial t}=\frac{\alpha_{s}(t)}{2 \pi} \int_{x}^{1} \frac{d z}{z} P_{q q}\left(\frac{x}{z}\right) p(z, t)
$$

one has to expand functions $p(x, t)$ into the series of the Chebyshev polynomials. In this way, the integration over $z$ in the evolution equation (4) can be performed, what leads to the system of linear differential equations:

$$
\frac{d p\left(x_{i}, t\right)}{d t}=\sum_{j=0}^{N-1} H_{i j} p\left(x_{j}, t\right)
$$


This system can be solved by using the standard Runge-Kutta method with initial conditions given by the input parametrisation $p\left(x_{j}, t_{0}\right)$. Truncated at $x_{0}$ moments are simply computed numerically via the integrating (3). The Chebyshev expansion provides a robust method of discretising a continuous problem. This allows computing the parton distributions for "not too singular" input parametrisation in the whole $x \in(0 ; 1)$ region. More detailed description of the Chebyshev polynomials method in the solving the QCD evolution equations is given e.g. in Appendix of [5].

\subsection{Evolution equations for truncated moments.}

LO DGLAP evolution equation for the truncated at $x_{0}$ Mellin moment of the nonsinglet structure function found by Forte, Magnea, Piccione and Ridolfi (FMPR) 4] has a form:

$$
\frac{\partial M[p]\left(x_{0}, n, t\right)}{\partial t}=\frac{\alpha_{s}(t)}{2 \pi} \int_{x_{0}}^{1} d y y^{n-1} p(y, t) G_{n}\left(\frac{x_{0}}{y}\right),
$$

where

$$
t \equiv \ln \frac{Q^{2}}{\Lambda_{Q C D}^{2}}
$$

and

$$
G_{n}(z) \equiv \int_{z}^{1} d y y^{n-1} P_{q q}(y)
$$

is the truncated anomalous dimension. Expanding $G_{n}\left(x_{0} / y\right)$ into Taylor series around $y=1$ gives

$$
\frac{\partial M[p]\left(x_{0}, n, t\right)}{\partial t}=\frac{\alpha_{s}(t)}{2 \pi} \sum_{j=0}^{m} C_{j n}^{(m)}\left(x_{0}\right) M[p]\left(x_{0}, n+j, t\right) .
$$

Eq. (9) is not diagonal but each $n$-th moment couples only with $(n+j)$-th $(j \geq 0)$ moments. Because the series of couplings to higher moments is convergent and furthermore the value of $(n+j)$-th moments decreases rapidly in comparison to the $n$-th moment, one can retain from (9) the closed system of $m+1$ equations:

$$
\begin{gathered}
\frac{\partial M[p]\left(x_{0}, n, t\right)}{\partial t}=\frac{\alpha_{s}(t)}{2 \pi} \\
\times \sum_{j=n}^{N_{0}+m} D_{n j}^{\left(N_{0}+m-n\right)}\left(x_{0}\right) M[p]\left(x_{0}, j, t\right) .
\end{gathered}
$$

Here

$$
N_{0} \leq n \leq N_{0}+m
$$

where $N_{0}$ is the lowest considered moment and $D$ is a triangular matrix. The solution of (10) has the form:

$$
\begin{gathered}
M[p]\left(x_{0}, n, t\right)=\sum_{k=n+1}^{N_{0}+m} A_{n k}\left(x_{0}\right) M[p]\left(x_{0}, k, t\right) \\
+\left(M[p]\left(x_{0}, n, t_{0}\right)-\sum_{k=n+1}^{N_{0}+m} A_{n k}\left(x_{0}\right) M[p]\left(x_{0}, k, t_{0}\right)\right) \\
\times \exp \left(c_{f} D_{n n}^{(m)}\left(x_{0}\right) \ln \frac{t}{t_{0}}\right) .
\end{gathered}
$$

Matrix elements $D_{i j}^{(m)}\left(x_{0}\right)$ and $A_{i j}\left(x_{0}\right)$ are given in 4 5]. In [5] results (12) have been compared to those, obtained with use of the Chebyshev-polynomial technique. The agreement of both approaches is excellent for higher moments $(n \geq 2)$ and not too large $x_{0} \leq 0.1$, even for a small number of terms $(m=4)$ in the truncated series (12). It also does not depend strongly on the scale $Q^{2}$ or the input parametrisation. In a case of the truncated first moment, a similar accuracy requires more terms $(m \geq 30)$ taken into account. In the next section we estimate the small- $x_{0}$ behaviour of the truncated first moment $M[p]\left(x_{0}, n=1, t\right)$.

\subsection{Small- $x_{0}$ behaviour of the truncated first moment.}

We would like to present a possible way of an approximate determination the small- $x_{0}$ behaviour of the truncated first moment $M[p]\left(x_{0}, 1, t\right)$. Our starting point is the evolution equation (6), which for the first moment has the form

$$
\frac{\partial q_{1}\left(x_{0}, t\right)}{\partial t}=\frac{\alpha_{s}(t)}{2 \pi} \int_{x_{0}}^{1} d y p(y, t) G_{1}\left(\frac{x_{0}}{y}\right) .
$$


Here we denote in short the truncated first moment by $q_{1}\left(x_{0}, t\right)$ :

$$
q_{j}\left(x_{0}, t\right) \equiv M[p]\left(x_{0}, j, Q^{2}\right) .
$$

Inserting $G_{1}(z)$ in the $\mathrm{LO}$ approximation:

$$
G_{1}(z)=\frac{8}{3} \ln (1-z)+\frac{4}{3}\left(z+\frac{z^{2}}{2}\right)
$$

we obtain

$$
\begin{gathered}
\frac{\partial q_{1}\left(x_{0}, t\right)}{\partial t}=\frac{2 \alpha_{s}(t)}{3 \pi}\left[x_{0} q_{0}\left(x_{0}, t\right)\right. \\
\left.+\frac{x_{0}^{2}}{2} q_{-1}\left(x_{0}, t\right)-2 \sum_{k=1}^{\infty} \frac{x_{0}^{k}}{k} q_{1-k}\left(x_{0}, t\right)\right] .
\end{gathered}
$$

In the above formula we have used the expansion of $\ln (1-z)$ :

$$
\ln (1-z)=-\sum_{k=1}^{\infty} \frac{z^{k}}{k}
$$

Taking into account that

$$
q_{j}\left(x_{0}, t\right)=x_{0}^{j-1} q_{1}\left(x_{0}, t\right)+(j-1) \int_{x_{0}}^{1} d y y^{j-2} q_{1}(y, t),
$$

we find

$$
\begin{gathered}
\frac{\partial q_{1}\left(x_{0}, t\right)}{\partial t}=\frac{2 \alpha_{s}(t)}{3 \pi}\left[q_{1}\left(x_{0}, t\right)\left(\frac{3}{2}-2 \sum_{k=1}^{\infty} \frac{1}{k}\right)\right. \\
\left.+\int_{x_{0}}^{1} d y\left(2 \sum_{k=1}^{\infty} \frac{x_{0}^{k}}{y^{k+1}}-\frac{x_{0}}{y^{2}}-\frac{x_{0}^{2}}{y^{3}}\right) q_{1}(y, t)\right] .
\end{gathered}
$$

The above result is LO DGLAP evolution equation for the truncated first moment of the nonsinglet parton distribution. This formula contains only $q_{1}$ and there is no mixing between $q_{1}$ and higher or lower moments $q_{j}$. Therefore we can adopt to (19) well known approach and write the evolution equation of $q_{1}$ in the moment space, what reads as follows:

$$
\frac{\partial M\left[q_{1}\right](n, t)}{\partial t}=\frac{2 \alpha_{s}(t)}{3 \pi} H(n) M\left[q_{1}\right](n, t)
$$

$H(n)$ is given by

$$
H(n)=2 \sum_{k=1}^{\infty}\left(\frac{1}{n+k}-\frac{1}{k}\right)+\frac{3}{2}-\frac{1}{n+1}-\frac{1}{n+2} .
$$

In this "moment of moment" approach (MM) we have obtained a simple equation for a some complicated structure $M\left[q_{1}\right](n, t)$, which is the (full) $n$-th moment of the truncated first moment of the parton density, namely

$$
M\left[q_{1}\right](n, t)=\int_{0}^{1} d x x^{n-1} \int_{x}^{1} d y p(y, t) .
$$

The solution of (20) is

$$
M\left[q_{1}\right](n, t)=M\left[q_{1}\right]\left(n, t_{0}\right) \exp [a(t) H(n)],
$$

where $M\left[q_{1}\right]\left(n, t_{0}\right)$ is a initial value of $M\left[q_{1}\right]$ for the low scale $t_{0}$ :

$$
M\left[q_{1}\right]\left(n, t_{0}\right)=\int_{0}^{1} d x x^{n-1} \int_{x}^{1} d y p\left(y, t_{0}\right)
$$

and

$$
a(t)=\frac{8}{33-2 N_{f}} \ln \frac{t}{t_{0}}
$$

with $N_{f}$ - the number of the quark flavours. Hence $q_{1}(x, t)$ is given by the inverse Mellin transform

$$
q_{1}(x, t)=\frac{1}{2 \pi i} \int_{c-i \infty}^{c+i \infty} d n x^{-n} M\left[q_{1}\right]\left(n, t_{0}\right) \exp [a(t) H(n)] .
$$


The behaviour of $q_{1}(x, t)$ for $x \rightarrow 0$ is governed by the leading (i.e. rightmost) singularities of $M\left[q_{1}\right]\left(n, t_{0}\right)$ as well as $H(n)$ in the $n$ complex plane [10. If we parametrise the input parton distribution $p\left(x, t_{0}\right)$ in the general form

$$
p\left(x, t_{0}\right) \sim x^{a_{1}}(1-x)^{a_{2}},
$$

we obtain

$$
\begin{aligned}
& M\left[q_{1}\right]\left(n, t_{0}\right) \sim \frac{1}{n} \beta\left(a_{1}+1, a_{2}+1\right)-\sum_{k=0}^{k_{\max }} \frac{(-1)^{k}}{k !} \\
& \times \frac{\Gamma\left(a_{2}+1\right)}{\Gamma\left(a_{2}+1-k\right)\left(a_{1}+1+k\right)\left(n+a_{1}+1+k\right)} .
\end{aligned}
$$

$\Gamma(z), \beta\left(z_{1}, z_{2}\right)$ in (28) are Euler functions and $k_{\max }$ depends on $a_{2}$ in the following way:

$$
k_{\max }= \begin{cases}\infty & \text { for fractional } a_{2}>0 \\ a_{2} & \text { for whole } a_{2} \geq 0\end{cases}
$$

One can see from (21) and (28) that $H(n)$ has the rightmost pole at $n=-1$, while $M\left[q_{1}\right]\left(n, t_{0}\right)$ at $n=0$ and $n=-a_{1}-1$. In this way, for the nonsingular at small- $x$ shape of the starting distribution $p\left(x, t_{0}\right)$ $\left(a_{1}=0\right)$, the simple pole at $n=0$ and the essential singularity at $n=-1$ are the leading ones. Then the small- $x_{0}$ behaviour of the truncated first moment can be determined by the method of steepest descent. We find

$$
\begin{aligned}
q_{1}(x, t) & \approx \frac{1}{a_{2}+1}-\sqrt{\frac{e}{2 \pi}} \beta\left(a_{2}+1, z\right) x_{0} z^{1.5}[z+2 a(t)]^{-0.5} \\
& \times \exp \left(a(t) H(z-1)+0.5 \sqrt{1-4 a(t) \ln \left(x_{0}\right)}\right),
\end{aligned}
$$

where

$$
z \equiv-\frac{1+\sqrt{1-4 a(t) \ln \left(x_{0}\right)}}{2 \ln \left(x_{0}\right)} .
$$

If we consider more singular input parametrisation $p\left(x, t_{0}\right)\left(a_{1}<0\right)$, this singular small- $x$ behaviour remains stable against LO $Q^{2} \mathrm{QCD}$ evolution. In this case the approximate behaviour of the truncated first moment $q_{1}(x, t)$ is governed by the leading simple poles of $M\left[q_{1}\right]\left(n, t_{0}\right)$, situated at $n=0$ and $n=-a_{1}-1$ :

$$
\begin{gathered}
q_{1}(x, t) \approx \beta\left(a_{1}+1, a_{2}+1\right)-\sum_{k=0}^{k_{\max }} \frac{(-1)^{k}}{k !} \\
\times \frac{\Gamma\left(a_{2}+1\right) x^{a_{1}+1+k}}{\Gamma\left(a_{2}+1-k\right)\left(a_{1}+1+k\right)} \exp \left[a(t) H\left(-a_{1}-1-k\right)\right] .
\end{gathered}
$$

In the next section we compare results (30)and 2.2

\section{Results for the truncated first moment within three differ- ent approaches.}

In this section we present numerical results for the truncated first moment of the nonsinglet parton distribution. Predictions obtained with use of different methods are denoted via CHEB, FMPR or MM, according to the applied approach (see Sect. 2.1 2.2 2.3 respectively).

Thus $q_{1}^{C H E B}\left(x_{0}, t\right)$ results from integrating

$$
q_{1}^{C H E B}\left(x_{0}, t\right)=\int_{x_{0}}^{1} d x x^{n-1} p^{C H E B}(x, t),
$$

where $p^{C H E B}(x, t)$ is the solution of (4)-(5), while $q_{1}^{F M P R}\left(x_{0}, t\right)$ is implied by (12) and has the form

$$
\begin{gathered}
q_{1}^{F M P R}\left(x_{0}, t\right)=\sum_{k=2}^{m+1} A_{1 k}\left(x_{0}\right) q_{k}\left(x_{0}, t\right) \\
+\left(q_{1}\left(x_{0}, t_{0}\right)-\sum_{k=2}^{m+1} A_{1 k}\left(x_{0}\right) q_{k}\left(x_{0}, t_{0}\right)\right) \\
\times \exp \left(c_{f} D_{11}^{(m)}\left(x_{0}\right) \ln \frac{t}{t_{0}}\right),
\end{gathered}
$$

where $q_{k}\left(x_{0}, t\right)$ is defined by (14).

Analytical approximate solutions (30)-(32), describing the low- $x_{0}$ behaviour of $q_{1}\left(x_{0}, t\right)$ within "moment 


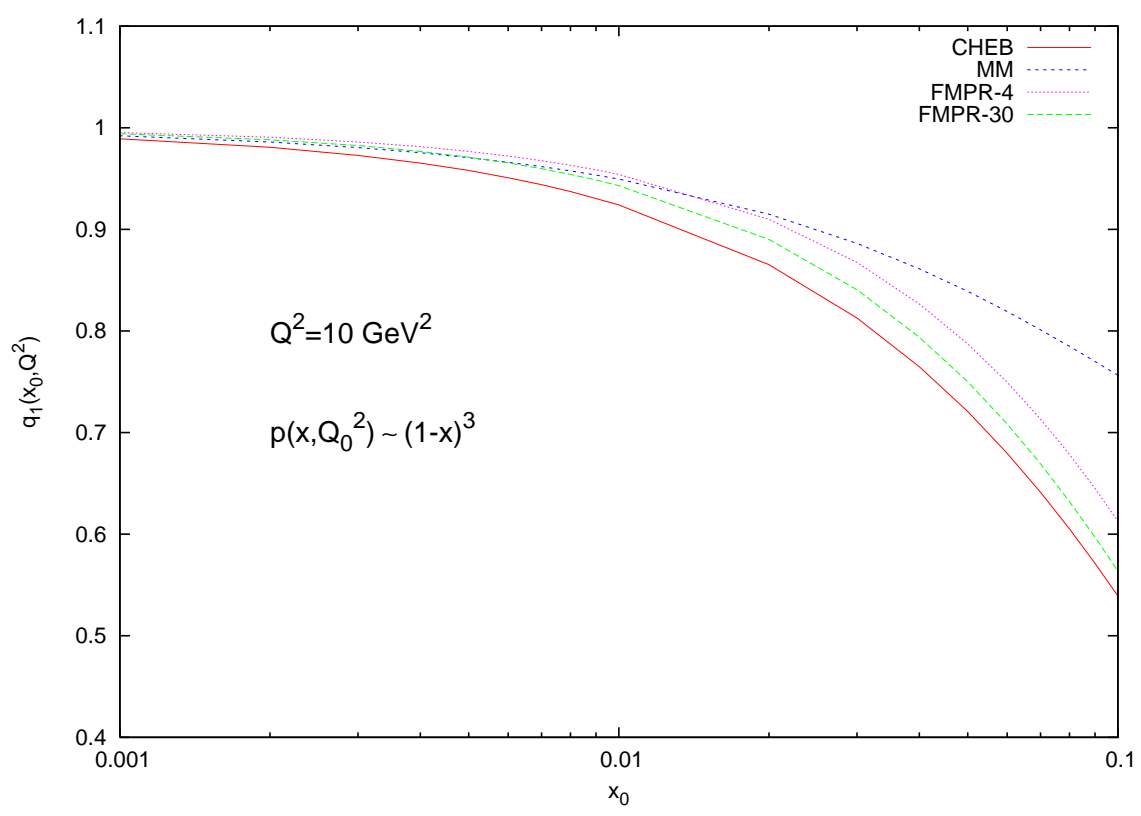

Figure 1: Small $x_{0}$ behaviour of the truncated at $x_{0}$ first moment of the nonsinglet spin structure function $g_{1}^{N S}$ in a case of the flat input (35). A comparison of (30) (MM) with the predictions based on the Chebyshev polynomials method (CHEB) and FMPR approach (34) for two values of the number of terms in the truncated series: $m=4, m=30$ is shown. The Bjorken Sum Rule is normalized to 1.

of moment" $\left(q_{1}^{M M}\right)$ approach are compared with $q_{1}^{C H E B}$ and $q_{1}^{F M P R}$ results in Figs.1-6. We consider two values of the number of terms in the truncated series for the FMPR-m predictions $(m=4, m=30)$. We show the results for the truncated at $x_{0}$ first moment of $g_{1}^{N S}$, which is a contribution to the Bjorken Sum Rule 2. In presented figures the total Bjorken Sum Rule is normalized to 1. We use two different inputs at $Q_{0}^{2}=1 \mathrm{GeV}^{2}$, namely:

$$
\begin{aligned}
& p\left(x, Q_{0}^{2}\right) \equiv g_{1}^{N S}\left(x, Q_{0}^{2}\right) \sim(1-x)^{3}, \\
& p\left(x, Q_{0}^{2}\right) \equiv g_{1}^{N S}\left(x, Q_{0}^{2}\right) \sim x^{-0.4}(1-x)^{2.5} .
\end{aligned}
$$

More singular at small- $x$ parametrisation (36) incorporates the latest knowledge about the low- $x$ behaviour of the polarised structure functions [1]. Truncated at $x_{0}=0.01$ integral $\int d x g_{1}^{N S}$ is reduced by about $8 \%$ for the Regge input (35) and about $20 \%$ for (36) in comparison to the total Bjorken Sum Rule. Figures 1-6 show that for small- $x_{0} \leq 0.01$ there is a good agreement between the MM results (30)-(32) and the predictions, obtained with use of the Chebyshev polynomial approach, which can be regarded as reliable. The percentage error

$$
E^{a}\left(x_{0}, Q^{2}\right)=\frac{\left|q_{1}^{a}\left(x_{0}, Q^{2}\right)-q_{1}^{C H E B}\left(x_{0}, Q^{2}\right)\right|}{q_{1}^{C H E B}\left(x_{0}, Q^{2}\right)} \cdot 100 \%,
$$

where $a$ denotes MM, FMPR-4 or FMPR-30 results, is about $3 \%$ in the case of MM solutions for $x_{0}=0.01$ and $Q^{2}=10 \mathrm{GeV}^{2}$, independently on the shape of the input parametrisation. Similar accuracy give taking into account more terms $(m=30)$ in the truncated series within FMPR approach (FMPR-30), while for $m=4$ the error (37) is about $4 \%$ in the case of the flat input and $6 \%$ in the case of the more singular one. $E^{a}\left(x_{0}, Q^{2}\right)$ grows slowly with increasing $Q^{2}$ (see Figs. 5-6) and for $Q^{2}=100 \mathrm{GeV}^{2}$ we get: $E^{M M}\left(x_{0}=\right.$ $\left.0.01, Q^{2}\right) \approx E^{F M P R-30}\left(x_{0}=0.01, Q^{2}\right) \approx 4 \%$ for the flat parametrisation and $7 \%$ respectively for the more singular input. Note, that for the truncation points $x_{0} \leq 0.01$ our approximate analytical solutions (30)-(32) are as reliable as the $F M P R-30$ predictions and more exact than the $F M P R-4$ results. This does not depend either on the shape of the input parametrisation nor on the value of $Q^{2}$.

In the next section we determine the low- $x$ contribution to the Bjorken Sum Rule.

\section{Low- $x$ contribution to the Bjorken Sum Rule.}

Among all moments of structure functions, the Bjorken Sum Rule (BSR) 2] is one of the convenient tests of QCD. BSR is a fundamental relation for polarised scattering, describing a relationship between spin dependent DIS and the weak coupling constant defined in neutron $\beta$-decay. In the limit of the infinite 


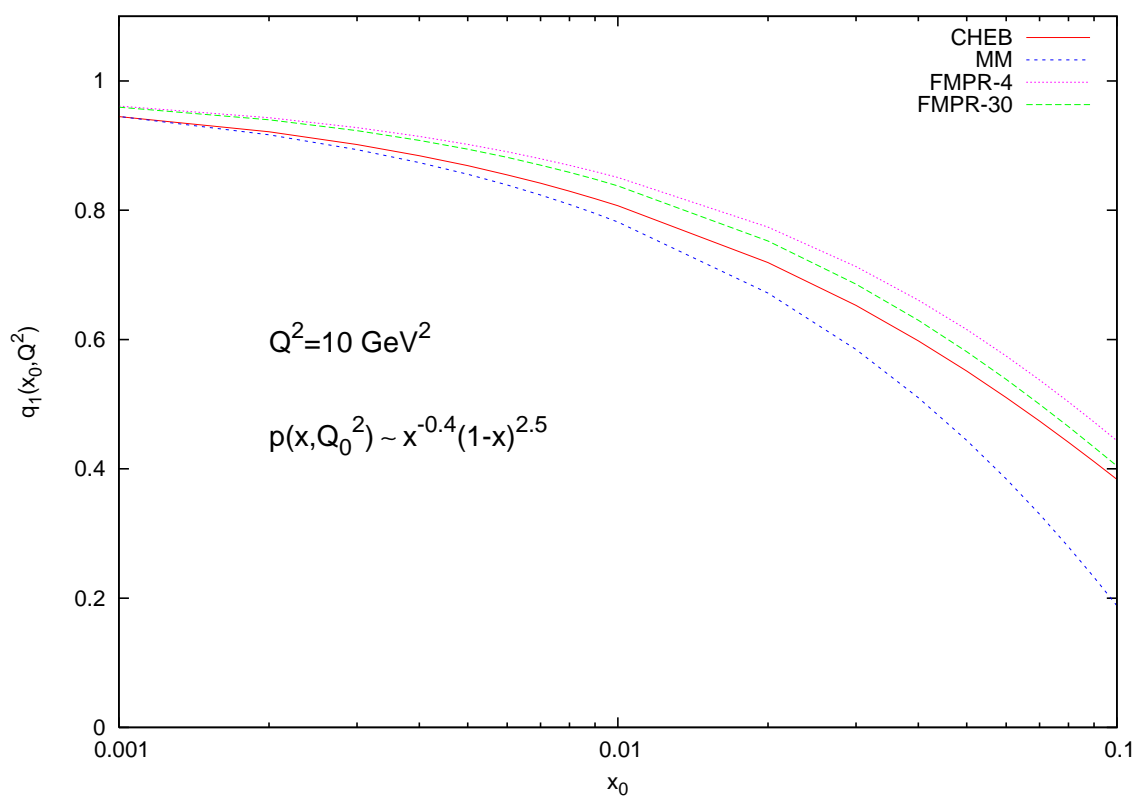

Figure 2: Small $x_{0}$ behaviour of the truncated at $x_{0}$ first moment of the nonsinglet spin structure function $g_{1}^{N S}$ in a case of the steep input (36). A comparison of (32) (MM) with the predictions based on the Chebyshev polynomials method (CHEB) and FMPR approach (34) for two values of the number of terms in the truncated series: $m=4, m=30$ is shown. The Bjorken Sum Rule is normalized to 1.

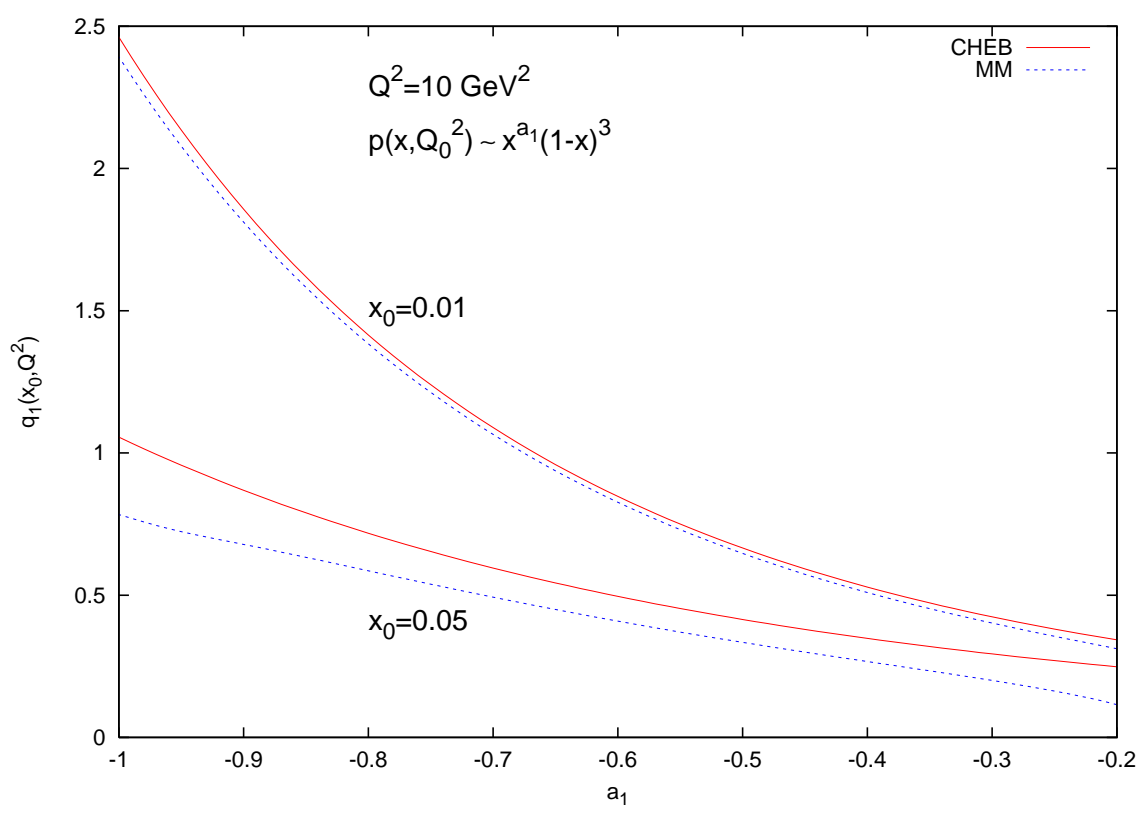

Figure 3: Truncated first moment $q_{1}^{M M}$ (32) as a function of $a_{1}$ in the input parametrisation $p\left(x, Q_{0}^{2}\right) \sim$ $x^{a_{1}}(1-x)^{3}$ for fixed $x_{0}=0.01$ and $x_{0}=0.05$. A comparison with the predictions based on the Chebyshev polynomials method is shown. 


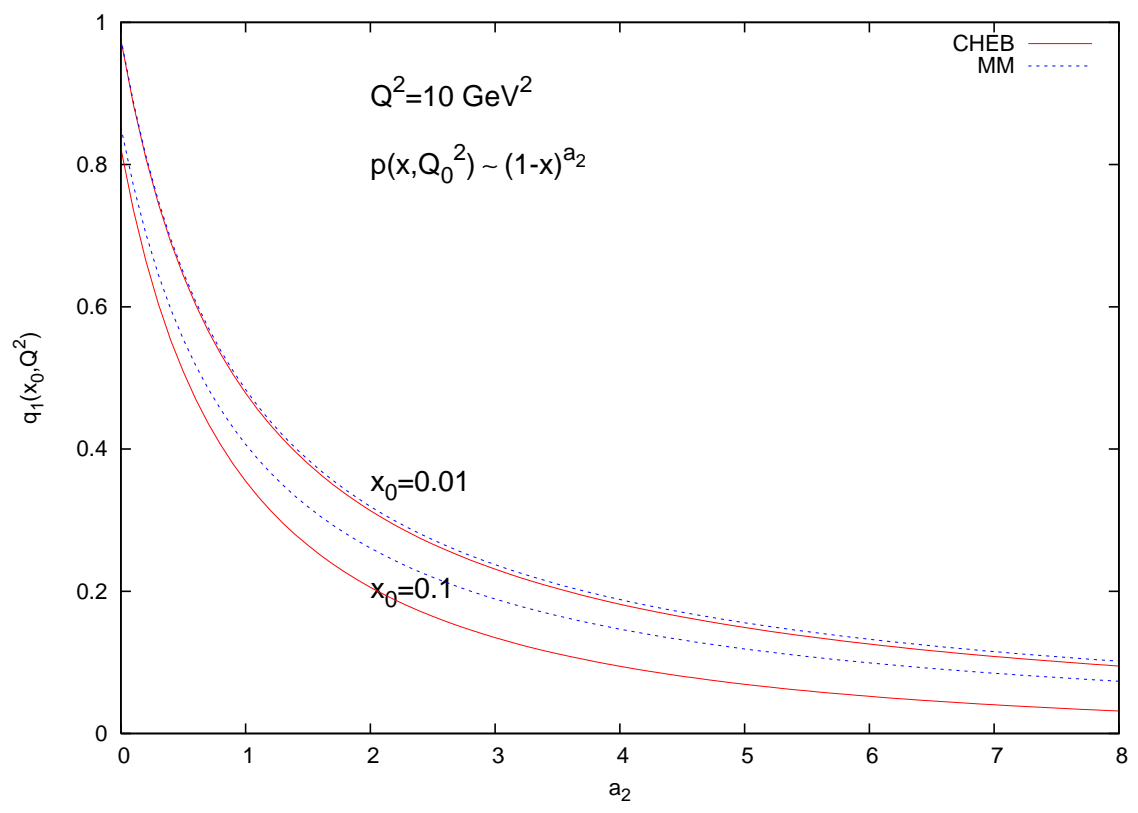

Figure 4: Truncated first moment $q_{1}^{M M}(30)$ as a function of $a_{2}$ in the input parametrisation $p\left(x, Q_{0}^{2}\right) \sim(1-x)^{a_{2}}$ for fixed $x_{0}=0.01$ and $x_{0}=0.1$. A comparison with the predictions based on the Chebyshev polynomials method is shown.

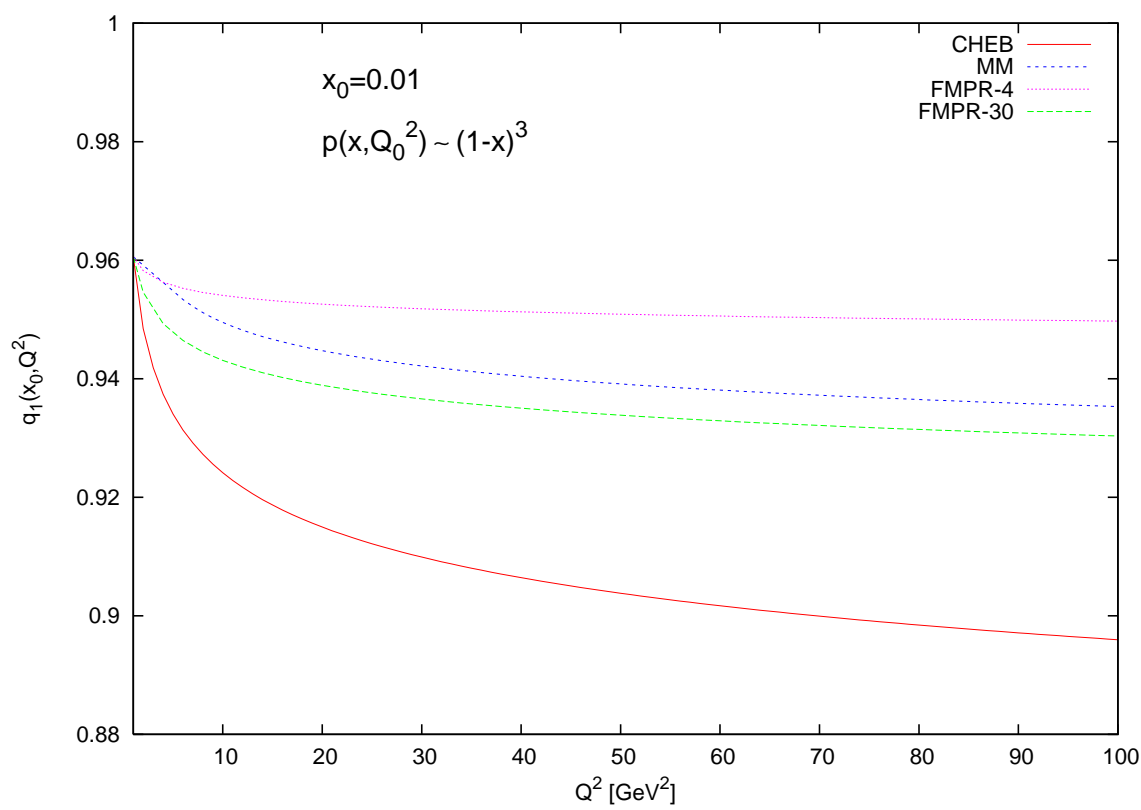

Figure 5: $Q^{2}$ dependence of the truncated first moment $q_{1}^{M M}$ (30) at fixed $x_{0}=0.01$ for input parametrisation (35). A comparison to the predictions based on the Chebyshev polynomials method and FMPR approach (34) for two values of the number of terms in the truncated series: $m=4, m=30$ is shown. 


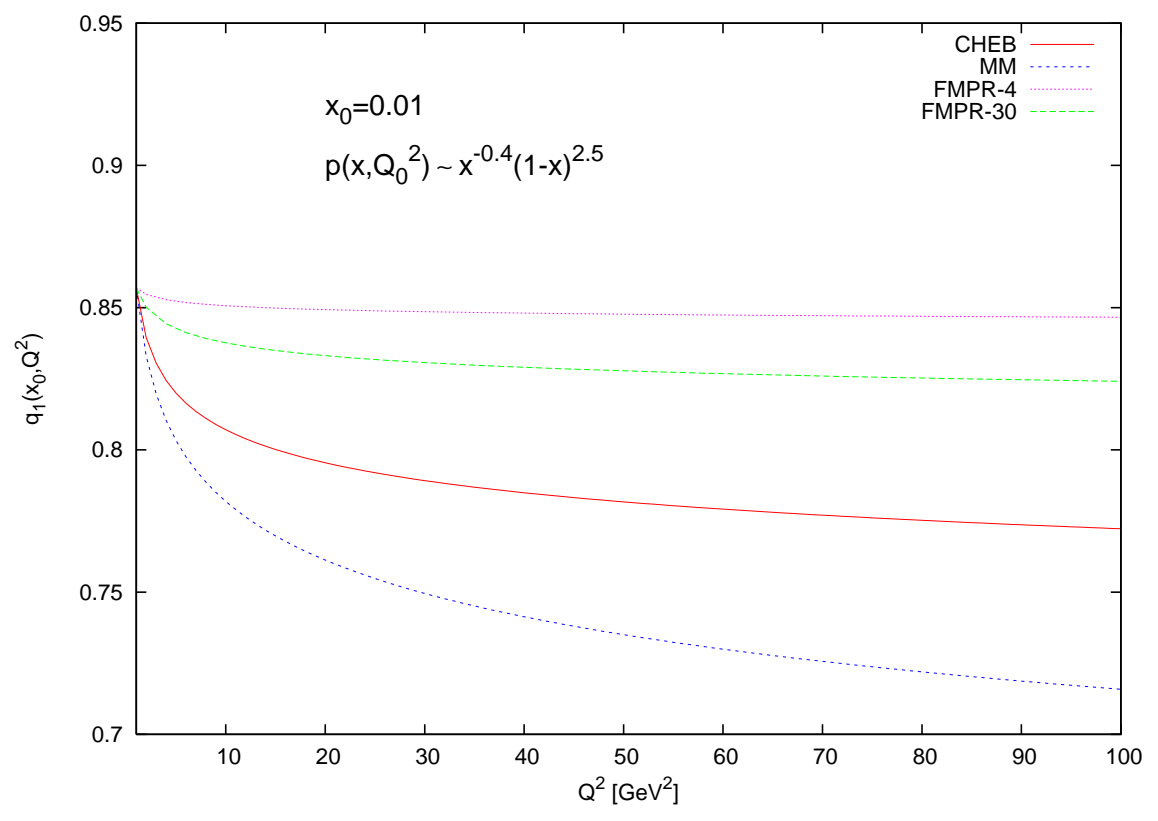

Figure 6: $Q^{2}$ dependence of the truncated first moment $q_{1}^{M M}$ (32) at fixed $x_{0}=0.01$ for input parametrisation (36). A comparison to the predictions based on the Chebyshev polynomials method and FMPR approach (34) for two values of the number of terms in the truncated series: $m=4, m=30$ is shown.

momentum transfer $Q^{2}$, the BSR reads:

$$
I_{B S R} \equiv \Gamma_{1}^{p}-\Gamma_{1}^{n}=\int_{0}^{1} d x\left(g_{1}^{p}(x)-g_{1}^{n}(x)\right)=\frac{1}{6} \frac{g_{A}}{g_{V}},
$$

where $g_{V}$ and $g_{A}$ are the vector and axial vector couplings. From recent measurements 12 $1.2695 \pm 0.0029$. BSR refers to the first moment of the nonsinglet spin dependent structure function $g_{1}^{N S}$ :

$$
g_{1}^{N S}\left(x, Q^{2}\right)=g_{1}^{p}\left(x, Q^{2}\right)-g_{1}^{n}\left(x, Q^{2}\right),
$$

where $g_{1}^{p}$ and $g_{1}^{n}$ are spin structure functions for proton and neutron. The asymptotic relation (38) at finite $Q^{2} \gg \Lambda_{Q C D}^{2}$ takes a form with pQCD corrections:

$$
\begin{gathered}
\int_{0}^{1} d x g_{1}^{N S}\left(x, Q^{2}\right)=\frac{1}{6} \frac{g_{A}}{g_{V}} \\
\times\left[1-\frac{\alpha_{s}}{\pi}-3.583\left(\frac{\alpha_{s}}{\pi}\right)^{2}-20.215\left(\frac{\alpha_{s}}{\pi}\right)^{3}\right] .
\end{gathered}
$$

The validity of the sum rule is confirmed in polarised DIS at the level of $10 \%$ 13, 14. Ewaluation of the sum rules requires knowledge of polarised structure functions over the entire region of $x$ : $0 \leq x \leq 1$. The experimentally accessible $x$ range for the spin dependent DIS is however limited $(0.7>x>0.003$ for SMC data [13, $0.6>x>0.023$ for HERMES data [15) and therefore one should extrapolate results to $x=0$ and $x=1$. The extrapolation to $x \rightarrow 0$, where structure functions grow strongly, is much more important than that to $x \rightarrow 1$, where structure functions vanish. The extrapolation towards $x=0$ suffers from large uncertainties, being essentially dependent on the used QCD fit. "Flexibility" of the chosen parametrisation appears in the agreement with the experimental data, giving however enough freedom in the unmeasured regions [16]. In a case of the BSR this allows for a significant reduction of the low- $x$ contribution.

In our approach we can test how the small- $x$ contribution to the BSR depends on the different (less or more steep) input parametrisations at the initial scale $Q_{0}^{2}=\mathrm{GeV}^{2}$ :

$$
g_{1}^{N S}\left(x, Q_{0}^{2}\right)=\eta x^{a_{1}}(1-x)^{a_{2}} .
$$

Here $\eta$ is a normalization factor:

$$
\eta=\frac{I_{B S R}}{\beta\left(a_{1}+1, a_{2}+1\right)}
$$




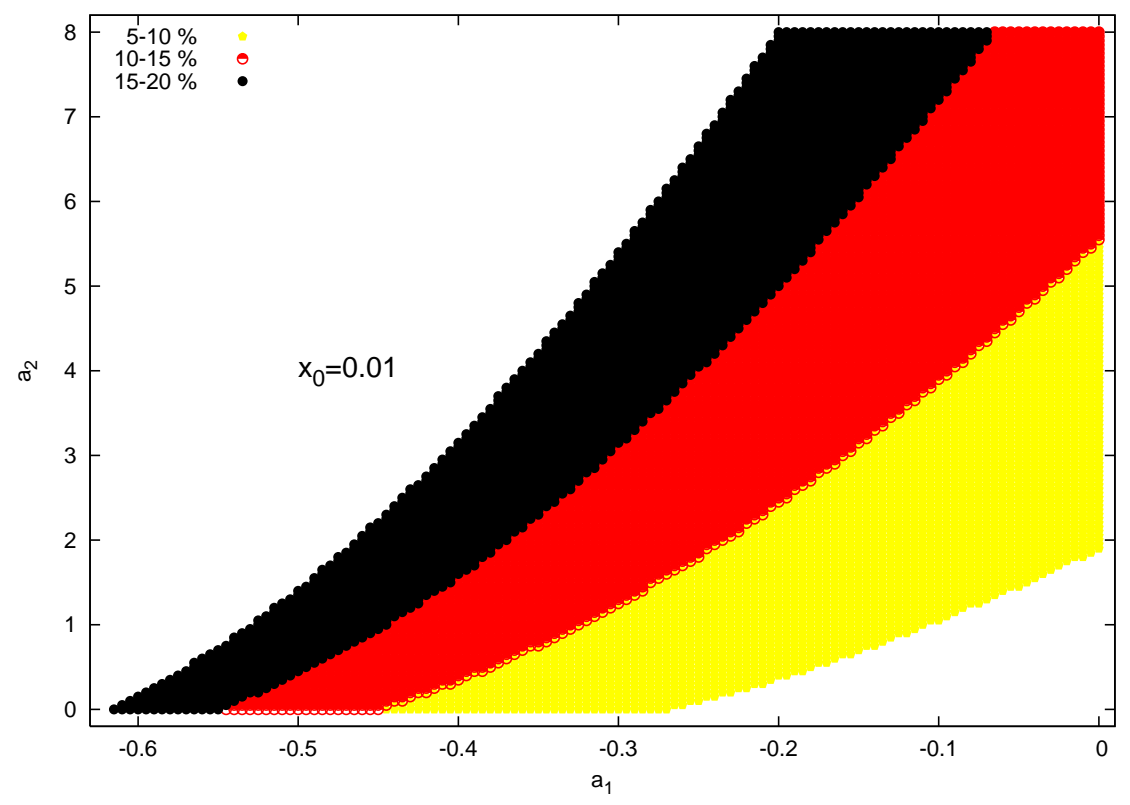

Figure 7: Low- $x$ contribution to the Bjorken Sum Rule (43) for different $a_{1}$ and $a_{2}$ in the input parametrisation (411). $x_{0}=0.01$ and $Q^{2}=5 \mathrm{GeV}^{2}$.

The exponent $a_{1}$ controls the behaviour of the structure function $g_{1}^{N S}$ as $x \rightarrow 0$ and the factor $(1-x)^{a_{2}}$ ensures the vanishing of $g_{1}^{N S}$ at $x \rightarrow 1$. The percentage contribution to the BSR, coming from small- $x$ region $0 \leq x \leq x_{0}$ is defined as:

$$
r\left(x_{0}, Q^{2}\right)=\frac{\int_{0}^{x_{0}} d x g_{1}^{N S}\left(x, Q^{2}\right)}{\int_{0}^{1} d x g_{1}^{N S}\left(x, Q^{2}\right)} \cdot 100 \%
$$

The ratio $r$ for $x_{0}=0.01$ varies from a few to tens percents for different configurations of $-1 \leq a_{1} \leq 0$ and $0 \leq a_{2} \leq 8$. The $r$-distribution at $x_{0}=0.01$ is shown in Fig.7. One can see that the small- $x$ contribution to the BSR grows with increasing $a_{2}$ and decreasing $a_{1}$. For the Regge flat parametrisation (35) $r$ is at the level of $5-10 \%$, what is significantly different from the result based on the input (36), where $r \sim 20 \%$. From theoretical analyses it is known that the small- $x$ behaviour of the nonsinglet polarised structure function $g_{1}^{N S}$ is governed by the double logarithmic terms i.e. $\left(\alpha_{s} \ln ^{2} x\right)^{n}$ 17, 18, 11. This leads to the singular at low- $x$ form of $g_{1}^{N S}$ :

$$
g_{1}^{N S}\left(x, Q^{2}\right) \sim x^{-\lambda}
$$

with $\lambda \approx 0.4$. LO DGLAP approach with use of the singular input (36) pretends the double logarithmic $l n^{2} x$ resummation. Low- $x$ experimental data 13 15] clearly confirm the rise of $g_{1}^{N S}$ in this region. However, the errors on the present data are too large to reliably support or contradict this $x^{-0.4}$ behaviour. "Freedom" in the initial parametrisation to satisfy the small- $x$ experimental extrapolation of the BSR is seen in Fig.8. The experimental data can be satisfactorily reproduced by e.g. nonsingular as $x \rightarrow 0$ input $\sim(1-x)^{6}$ and by e.g. the singular one $\sim x^{-0.5}(1-x)^{1}$ as well. SMC and SLAC measurements imply that $10-20 \%$ of the BSR comes from $x$ values less than 0.01 [13, 14] 19. Also HERMES data 15] enable to determine the low- $x$ contribution to the BSR at 0.023 between $20-40 \%$. Wide range of these estimations could be restricted by new spin data concerning this mystery and interesting small- $x$ region.

\section{Conclusions.}

In this paper we have compared results for the truncated at $x_{0}$ first moment $q_{1}$ of the parton distribution obtained within different approaches. Thus we have solved numerically LO DGLAP evolution equation for the nonsinglet function in the $x$-space using Chebyshev polynomial expansion and then after integrating over $x$ we have got the prediction $q_{1}^{C H E B}$, which can be treated as an exact one. Next, using evolution equations written in the moment space, we have found the closed system of $m+1$ solutions for truncated moments, where obtained $q_{1}^{F M P R-m}$ result is expressed by values of the $m$ higher moments. Considered number of terms in the truncated series was $m=4$ and $m=30$. Working in the moment space we have also found an alternative way to determine the small- $x_{0}$ behaviour of the truncated first moment. Taking into account the relation between $n$-th and $j$-th truncated moment, we were able to derive the evolution equation, which 


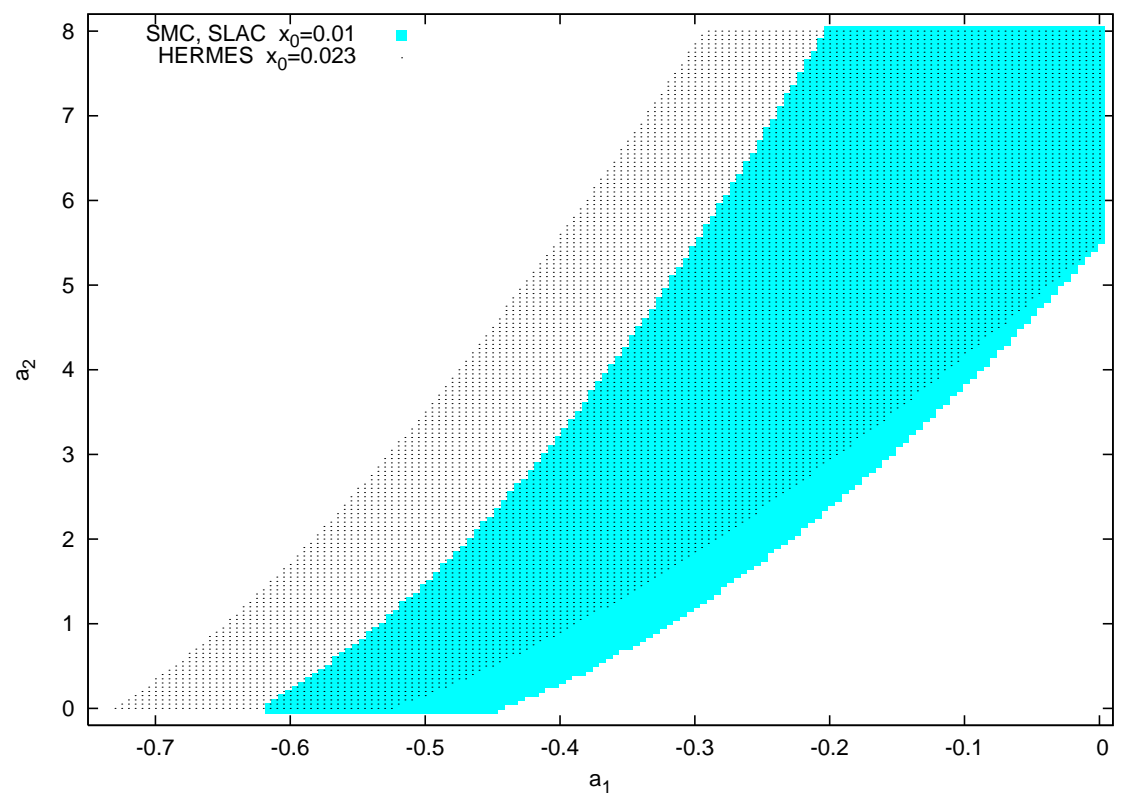

Figure 8: Constraints on the parametrisation of $g_{1}^{N S}$, implied by the experimental estimations of the low- $x$ contribution to the Bjorken Sum Rule. SMC, SLAC: $10 \% \leq r\left(x_{0}=0.01,5\right) \leq 20 \%$ (full colour), HERMES: $20 \% \leq r\left(x_{0}=0.023,5\right) \leq 40 \%$ (dotted).

does not contain mixing between different moments. Then, adopting the standard analytical method of full moments to the case of the first truncated moment, we have found approximate behaviour of $q_{1}$ as $x \rightarrow 0$. In this way the inverse Mellin transform performed with use of the method of steepest descent implies the result $q_{1}^{M M}$ within our modified "moment of moment" approach.

We have shown that for small- $x_{0} \leq 0.01$ there is a good agreement between the MM results and the reliable predictions, obtained with use of the Chebyshev polynomial method. This agreement occurs independently either on the shape of the input parametrisation or on the value of $Q^{2}$. It has been also found, that for small $x_{0}$ the accuracy of $q_{1}^{M M}$ and $q_{1}^{F M P R-30}$ results are similar, being clearly better than in the case of $q_{1}^{F M P R-4}$ predictions.

We have presented results concerning the spin structure function $g_{1}^{N S}$ and the truncated at $x_{0}=0.01$ contribution to the Bjorken Sum Rule. It has been found, that the choice of the input parametrisation has a large impact on the evaluation of the low- $x$ contribution to the BSR. This contribution can vary from a few percents for the flat $(\sim$ const $)$ input to tens percents for the steep $\left(\sim x^{-0.5}\right)$ one. Recent experimental data confirm the rise of the polarised structure functions at small- $x$. However, because of the large uncertainties, reliable support or contradiction of the theoretical expectations is still out of reach.

\section{References}

[1] K. Gottfried, Phys. Rev. Lett. 18, (1967) 1174.

[2] J.D. Bjorken, Phys. Rev. 148, (1966) 1467; Phys. Rev. D1, (1970) 1376.

[3] V.N. Gribov and L.N. Lipatov, Sov. J. Nucl. Phys. 15, (1972) 438-450 and 675-684.

Yu.L. Dokshitzer, Sov. Phys. JETP 46, (1977) 641-653.

G. Altarelli, and G. Parisi, Nucl. Phys. B126, (1977) 298.

[4] S. Forte and L. Magnea, Phys. Lett. B448, (1999) 295-302 arXiv:hep-ph/9812479.

S. Forte, L. Magnea, A. Piccione and G. Ridolfi, Nucl. Phys. B594, (2001) 46-70 arXiv:hep-ph/0006273.

A.Piccione, Phys. Lett. B518, (2001) 207-213 arXiv:hep-ph/0107108.

[5] D. Kotlorz and A. Kotlorz, Acta Phys. Pol. B36, (2005) 3023-3039 arXiv:hep-ph/0510295.

[6] S. Kumano and T.-H. Nagai, J. Comput. Phys. 201, (2004) 651-664 arXiv:hep-ph/0405160.

[7] C. Coriano and C.Savkli Comput. Phys. Commun. 118, (1999) 236-258 arXiv:hep-ph/9803336.

R. Kobayashi, M. Konuma, S. Kumano and M. Miyama, Prog. Theor. Phys. Suppl. 120, (1995) 257-262 arXiv:hep-ph/9412341.

[8] J. Kwieciński and D. Strózik-Kotlorz, Z. Phys. C48, (1990) 315-326.

J. Kwieciński and B. Ziaja, Phys. Rev. D60, (1999) 054004 arXiv:hep-ph/9902440. 
B. Badełek and J. Kwieciński, Phys. Lett. B418, (1988) 229-236 arXiv:hep-ph/9709363.

J. Kwieciński and M. Maul, Phys. Rev. D67, (2003) 034014 arXiv:hep-ph/0111031.

[9] S.E. El-gendi, Comput. J. 12, (1969) 282-287.

[10] A. De Rújula, S.L. Glashow, H.D. Politzer, S.B. Treiman, F. Wilczek and A. Zee, Phys. Rev. D10, (1974) 1649-1652.

[11] B.I. Ermolaev, M. Greco, S.I. Troyan, Nucl. Phys. B571, (2000) 137-150 arXiv:hep-ph/9906276;

Nucl. Phys. B594, (2001) 71-88 arXiv:hep-ph/0009037.

[12] The Particle Data Group: S. Eidelman et al., Phys. Lett. B592, (2004) 1.

[13] SMC Collaboration: D. Adams et al., Phys. Rev. D56, (1997) 5330;

B. Adeva et al., Phys. Lett.B420, (1998) 180;

Phys. Rev. D58, (1998) 112002.

[14] E154 Collaboration: K. Abe et al., Phys. Rev. Lett. 79, (1997) 26.

E155 Collaboration: P.L. Anthony et al., Phys. Lett. B463, (1999) 339.

[15] HERMES Collaboration: K. Ackerstaff et al., Phys. Lett.B404, (1997) 383;

Phys. Lett.B464, (1999) 123;

A. Airapetian et al., Phys. Lett.B442, (1998) 484.

[16] HERMES Collaboration: A. Airapetian et al., Phys. Rev.D71, (2005) 012003 arXiv:hep-ex/0407032.

[17] J. Kwieciński, Acta Phys. Pol. B27, (1996) 893.

[18] J. Bartels, B.I. Ermolaev, M.G. Ryskin, Z. Phys. C70, (1996) 273;

Z. Phys. C72, (1996) 627.

[19] S.D. Bass, Rev. Mod. Phys. Pol. 77, (2005) 1257 arXiv:hep-ph/0411005. 